\title{
Measurement of the Isoscalar Monopole Response in the Neutron-Rich Nucleus ${ }^{68} \mathrm{Ni}$
}

\author{
M. Vandebrouck, ${ }^{1,2,{ }^{*}}$ J. Gibelin, ${ }^{2}$ E. Khan, ${ }^{1}$ N. L. Achouri, ${ }^{2}$ H. Baba, ${ }^{4}$ D. Beaumel, ${ }^{1}$ Y. Blumenfeld, ${ }^{1}$ M. Caamaño, ${ }^{5}$ \\ L. Càceres, ${ }^{3}$ G. Colò, ${ }^{6}$ F. Delaunay, ${ }^{2}$ B. Fernandez-Dominguez, ${ }^{5}$ U. Garg, ${ }^{7}$ G. F. Grinyer, ${ }^{3}$ M. N. Harakeh, ${ }^{8,3}$ \\ N. Kalantar-Nayestanaki, ${ }^{8}$ N. Keeley, ${ }^{9}$ W. Mittig, ${ }^{10}$ J. Pancin, ${ }^{3}$ R. Raabe, ${ }^{11}$ T. Roger,${ }^{11,3}$ P. Roussel-Chomaz, ${ }^{12}$ H. Savajols, ${ }^{3}$ \\ O. Sorlin, ${ }^{3}$ C. Stodel, ${ }^{3}$ D. Suzuki, ${ }^{10,1}$ and J. C. Thomas ${ }^{3}$ \\ ${ }^{1}$ IPN Orsay, Université Paris Sud, IN2P3-CNRS, F-91406 Orsay Cedex, France \\ ${ }^{2}$ LPC Caen, ENSICAEN, Université de Caen, CNRS/IN2P3, F-14050 CAEN Cedex, France \\ ${ }^{3}$ Grand Accélérateur National d'Ions Lourds (GANIL), CEA/DSM-CNRS/IN2P3, Bvd Henri Becquerel, 14076 Caen, France \\ ${ }^{4}$ RIKEN Nishina Center, 2-1 Hirosawa, Wako, Saitama 351-0198, Japan \\ ${ }^{5}$ Universidade de Santiago de Compostela, E-15706 Santiago de Compostela, Spain \\ ${ }^{6}$ Dipartimento de Fisica Università degli Studi di Milano and INFN, Sezione di Milano, 20133 Milano, Italy \\ ${ }^{7}$ Physics Department, University of Notre Dame, Notre Dame, Indiana 46556, USA \\ ${ }^{8}$ KVI-CART, University of Groningen, NL-9747 AA Groningen, The Netherlands \\ ${ }^{9}$ National Centre for Nuclear Research ul. Andrzeja Sot tana 7, 05-400 Otwock, Poland \\ ${ }^{10}$ NSCL, Michigan State University, East Lansing, Michigan 48824-1321, USA \\ ${ }^{11}$ Instituut voor Kern- en Stralingsfysica, KU Leuven, B-3001 Leuven, Belgium \\ ${ }^{12}$ CEA-Saclay DSM, F-91191 Gif sur Yvette Cedex, France
}

(Received 12 March 2014; published 16 July 2014; publisher error corrected 18 July 2014)

\begin{abstract}
The isoscalar monopole response has been measured in the unstable nucleus ${ }^{68} \mathrm{Ni}$ using inelastic alpha scattering at $50 \mathrm{~A} \mathrm{MeV}$ in inverse kinematics with the active target MAYA at GANIL. The isoscalar giant monopole resonance (ISGMR) centroid was determined to be $21.1 \pm 1.9 \mathrm{MeV}$ and indications for a soft monopole mode are provided for the first time at $12.9 \pm 1.0 \mathrm{MeV}$. Analysis of the corresponding angular distributions using distorted-wave-born approximation with random-phase approximation transition densities indicates that the $L=0$ multipolarity dominates the cross section for the ISGMR and significantly contributes to the low-energy mode. The $L=0$ part of this low-energy mode, the soft monopole mode, is dominated by neutron excitations. This demonstrates the relevance of inelastic alpha scattering in inverse kinematics in order to probe both the ISGMR and isoscalar soft modes in neutron-rich nuclei.
\end{abstract}

DOI: 10.1103/PhysRevLett.113.032504

Measurements in exotic nuclei have yielded an abundance of new information in recent years. For instance, a more general view on magicity has emerged in the past decade [1]. It has also been suggested that measurements of giant resonances in exotic nuclei could help clarify another important problem related to nuclear incompressibility [2]: despite significant progress in our understanding of the nuclear incompressibility, one cannot converge towards a value that is more accurate than the 10\%-20\% level. In other words, it seems difficult to single out an energy density functional that can give a comprehensive description of the ISGMR, especially in keeping with new data on isotopic chains including open-shell systems and on neutron-rich nuclei [3-6]. This can be seen in the recent theoretical works on the subject [7-12]. The measurement of the ISGMR in exotic neutron-rich nuclei is, therefore, of paramount importance. Such measurements in exotic nuclei would significantly improve our understanding of nuclear incompressibility as was the case for nuclear magicity in the past decade. In addition, a soft monopole mode has been predicted around $14 \mathrm{MeV}$ in neutron-rich nuclei by several relativistic and nonrelativistic models [13-15], but
PACS numbers: 21.10.Re, 21.60.Jz, 24.30.Cz, 24.50.+g

it has never been observed, due to the difficulty in measuring the monopole response in nuclei far from stability. The soft monopole mode is predicted to be noncollective and its observation could bring valuable information on spin-orbit splitting [16]. It is therefore essential to measure the isoscalar (in-phase motion of protons and neutrons) monopole response in neutron-rich nuclei.

The measurement of giant resonances in unstable nuclei is a particularly challenging task and has, until now, been mainly limited to the isovector (protons and neutrons out of phase) giant dipole resonance in neutron-rich radioactive $\mathrm{O}$, $\mathrm{Ne}, \mathrm{Sn}$ isotopes, and in ${ }^{68} \mathrm{Ni}$ [17]. Such measurements provided the first evidence for soft isovector dipole modes in unstable nuclei. These modes are often reanalyzed, showing a nontrivial pattern, departing from the simple pygmy resonance picture of a soft oscillation of a neutron skin around a proton-neutron core as they can have mixed isovector and isoscalar components [18]. In the case of the isoscalar response, only one measurement has thus far been performed on an unstable nucleus. The $N=Z$ nucleus ${ }^{56} \mathrm{Ni}$, demonstrated a behavior of the monopole and quadrupole responses similar to neighbouring stable nuclei 
such as ${ }^{58} \mathrm{Ni}$ [4]. Evidence for a soft isovector dipole strength in ${ }^{68} \mathrm{Ni}[19,20]$, the prediction of a soft monopole strength in neutron-rich nuclei [14], as well as the possibility for a strong isoscalar component of the soft dipole mode [18], set ${ }^{68} \mathrm{Ni}$ at the center of interest to measuring the isoscalar response in neutron-rich nuclei.

The best probe for the investigation of the ISGMR is inelastic alpha or deuteron scattering at energies of a few hundred MeV [2]. Due to the higher cross section [2] of the ${ }^{68} \mathrm{Ni}\left(\alpha, \alpha^{\prime}\right)^{68} \mathrm{Ni}^{*}$ reaction and the absence of unwanted background due to deuteron breakup, the alpha probe is more suited. However, the kinematics of the ${ }^{68} \mathrm{Ni}\left(\alpha, \alpha^{\prime}\right)^{68} \mathrm{Ni}^{*}$ reaction is such that the recoiling alpha has less energy than a recoiling deuteron, and as a consequence is more difficult to detect. Only one experiment, using a deuteron probe, has been performed for unstable nuclei up to now, due to the difficult experimental conditions inherent to the use of a radioactive beam [4]. The ISGMR cross section is peaked at $0^{\circ}$ in the center-of-mass (c.m.) frame, which corresponds to very low energies of the recoiling particle in the laboratory frame. In order to cover the excitation energy range from 0 to $30 \mathrm{MeV}$, between $0^{\circ}$ and $8^{\circ}$ in the c.m. frame, it is necessary to measure recoiling alpha particles with energies between $300 \mathrm{keV}$ and $4 \mathrm{MeV}$ at angles from $0^{\circ}$ to $90^{\circ}$ in the laboratory frame. This experimental challenge can not be met with a standard target and recoiling-particle telescopes due to their relatively high energy detection thresholds and energy straggling in the target. An active target, where a gas volume is used as both a thick reaction target and as the detector itself, provides an increase of more than two orders of magnitude on the number of scattering atoms compared to a standard solid target, while minimizing straggling effects. In this Letter, we report on the first inelastic alpha scattering measurement of the isoscalar monopole response for an unstable neutron-rich nucleus, ${ }^{68} \mathrm{Ni}$.

A secondary ${ }^{68} \mathrm{Ni}$ beam at $50 \mathrm{~A} \mathrm{MeV}$ was produced at the GANIL facility from the fragmentation of a ${ }^{70} \mathrm{Zn}$ primary beam at $62.3 A \mathrm{MeV}$ on a $29 \mathrm{mg} / \mathrm{cm}^{2}{ }^{9} \mathrm{Be}$ target and located at the entrance of the LISE [21] spectrometer. A $560 \mu \mathrm{m}$ achromatic degrader was used for purification. The average beam intensity of ${ }^{68} \mathrm{Ni}$ was $4 \times 10^{4} \mathrm{pps}$ with a purity of $75 \% \pm 6 \%$. With respect to the experimental constraints mentioned above an active target is the key to measuring the isoscalar monopole response in unstable nuclei. The active target MAYA, developed at GANIL [22] for nuclear physics experiments, is a time projection chamber (TPC) with an active volume of a $28 \times 25 \times$ $20 \mathrm{~cm}^{3}$. Electrons produced from the ionization of the gas by charged particles traversing its volume drift in an applied electric field to a set of 32 amplification wires that are parallel to the beam direction. For a two-body reaction, scattered and recoiling particles are in a plane which can be determined using the drift time required by the electrons to reach the wires. The avalanches on the wires induce signals on a matrix of $32 \times 32$ hexagonal pads connected to
GASSIPLEX [22] chips. MAYA was filled with He gas, at a pressure of 500 mbar (with $5 \% \mathrm{CF}_{4}$ used as a quenching gas) and was positioned at the end of the LISE beam line. An electrostatic mask [23] was placed below the beam trajectory in MAYA. The role of this mask was to reduce the charges due to the highly-ionizing beam particles in comparison to those induced by the recoiling particles, thus effectively increasing the dynamic range. For normalization purposes, the incident beam intensity was measured by recording the number of counts recorded on the central wire.

For each event, two physical observables are required to reconstruct the kinematics of the reaction: the energy and the angle of the recoiling alpha particle in the laboratory frame. The projected recoil angle in the horizontal plane is reconstructed using the "global fitting method" described in [24], in which a straight-line trajectory is determined by minimizing the orthogonal distance of the center of the pads weighted by their charge to the line. The intersection of the trajectories of the alpha particle and the beam particle determines the vertex of the reaction projected on the pad plane. Between this vertex and the end of the recoil track, all charges are projected on the direction of the recoil trajectory, thereby defining the position of the Bragg peak and thus the range in two dimensions. The third dimension is deduced from the drift times of the electrons towards the anode wires. The recoil angle with respect to the beam axis and the range in the He gas are thus determined on an eventby-event basis. The energy of the alpha particle is deduced from its range in the gas [25]. A reliable trajectory reconstruction is achieved for alpha particles having energies higher than $600 \mathrm{keV}$. The three dimensional reconstruction of scattering trajectories in MAYA provides the kinematics of the ${ }^{68} \mathrm{Ni}\left(\alpha, \alpha^{\prime}\right)$ reaction as shown in Fig. 1(a).

The data were then transformed into the c.m. frame using two-body relativistic kinematics and corrected for geometrical and reconstruction, including the detection part, efficiencies of the detector [Fig. 1(b)]. These efficiencies were deduced by simulation using the GEANT4-based [26] ACTARsim package. This package has been validated using detailed comparisons between simulated and measured alpha particle trajectories [27]. The corresponding projected excitation-energy spectrum of ${ }^{68} \mathrm{Ni}$ is displayed in Fig. 2. This spectrum displays a peak centered at $0 \mathrm{MeV}$ corresponding to elastic scattering and to inelastic scattering to low-lying states. For the elastic peak, the missing mass resolution FWHM is $2 \mathrm{MeV}$. This is dominated by uncertainties associated with the trajectory reconstruction. At energies between 12 and $30 \mathrm{MeV}$, peaks in the excitation-energy spectrum point towards the presence of several isoscalar modes. As expected, the relative strength of the observed isoscalar modes strongly depends on the scattering angle. Figure 2 displays the excitation-energy spectrum for all angles (a) and at $\theta_{\text {c.m. }}=5.5^{\circ}(\mathrm{b})$, highlighting the emergence of a low-energy mode at $\sim 13 \mathrm{MeV}$. 

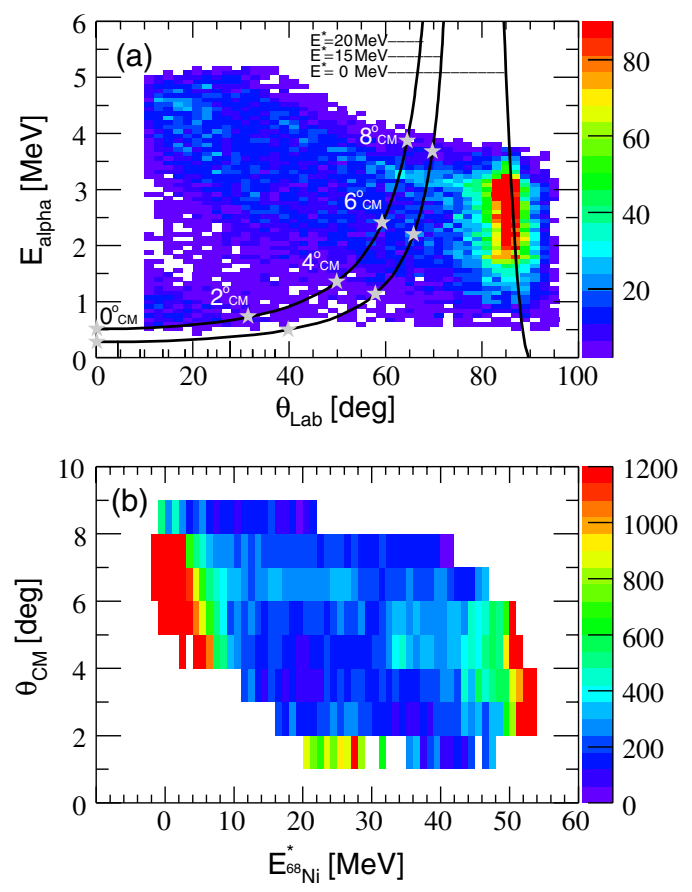

FIG. 1 (color online). (a) Scatter plot of recoiling alpha energy versus scattering angle in the laboratory frame for the ${ }^{68} \mathrm{Ni}$ beam. (b) Corresponding events transformed in the c.m. frame, and corrected for the geometrical and reconstruction efficiencies.

The data analysis was first performed by fitting the excitation energy spectra at different angles below $\theta_{\text {c.m. }}=9^{\circ}$, with the flat background and four Lorentzians corresponding to the observed peaks around 13, 16, 21, and $26 \mathrm{MeV}$, respectively. The widths of the Lorentzians were left free in the fit. With this method we have identified three resonances at $12.9 \pm 1.0,15.9 \pm 1.3$, and $21.1 \pm 1.9 \mathrm{MeV}$ with a width of $1.2 \pm 0.4,2.3 \pm 1.0$, and $1.3 \pm 1.0 \mathrm{MeV}$, respectively. Above $23 \mathrm{MeV}$, the broad dotted distribution corresponds to additional multipolarities such as $L=0,1,3$, and to the well-known fragmentation of giant resonances in these medium-mass nuclei $[2,28]$. Considering the present statistics, it is not relevant to attempt an analysis of this highenergy part of the spectrum which is already difficult to interpret in the case of stable nuclei and high statistics. The angular distributions of the Lorentzians at 12.9 and 21.1 MeV are displayed in Figs. 3(a) and 3(b), and are well reproduced by distorted-wave-born approximation (DWBA) calculations assuming an $L=0$ multipolarity. Nevertheless, as the angular distributions are less characteristic in the $\left[\theta_{\text {c.m. }}=4.5^{\circ}, \theta_{\text {c.m. }}=8.5^{\circ}\right]$ region, $L=1$ and $L=2$ contributions are possible for the low-energy mode, and the present analysis does not allow us to disentangle them. The theoretical angular distributions were calculated with the code FRESCO [29]. Real and imaginary diagonal and transition potentials were determined semimicroscopically with the code DFPOT [30] using the single-folding model and calculated ${ }^{68} \mathrm{Ni}$ densities and a Gaussian alpha-nucleon interaction. The parameters of the alpha-nucleon interaction

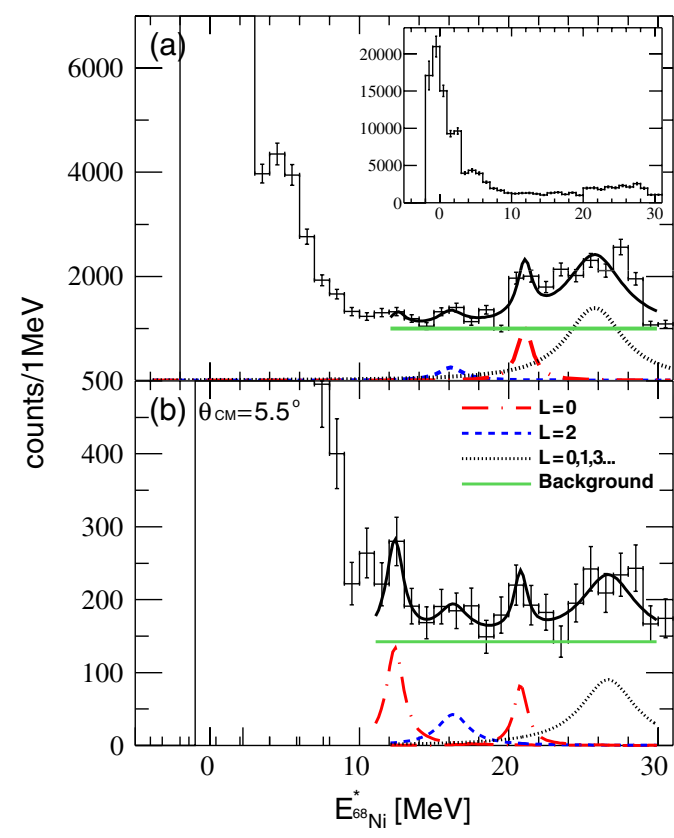

FIG. 2 (color online). (a) ${ }^{68} \mathrm{Ni}$ excitation-energy spectrum for all angles deduced from the alpha recoil kinematics and corrected for geometrical and reconstruction efficiencies. (b) Same for $\theta_{\text {c.m. }}=5.5^{\circ}$. For both spectra, the subtracted background is indicated by the horizontal green solid line. The data were fit with Lorentzians at $12.9 \mathrm{MeV}$ (red dot-dashed line), $15.9 \mathrm{MeV}$ (blue short-dashed line) and $21.1 \mathrm{MeV}$ (red dot-dashed line) for the low-energy mode, the isoscalar giant quadrupole resonance (ISGQR) and the ISGMR, respectively (see text). Statistical uncertainty and the estimated uncertainty on the efficiency correction are taken into account.

were obtained by fitting the ${ }^{64} \mathrm{Ni}(\alpha, \alpha)$ elastic scattering data at $43 \mathrm{~A} \mathrm{MeV} \mathrm{[31].} \mathrm{Transition} \mathrm{densities} \mathrm{for}{ }^{68} \mathrm{Ni}$ were calculated with microscopic random-phase approximation (RPA) [32], they were obtained self consistently from the same Skyrme functional as in the Hartree-Fock calculation, namely the SkI2 $\left(K_{\infty}=241 \mathrm{MeV}\right)[33]$ interaction.

To check the consistency of these results, another independent analysis was performed using the multipoledecomposition analysis (MDA). The transition potentials

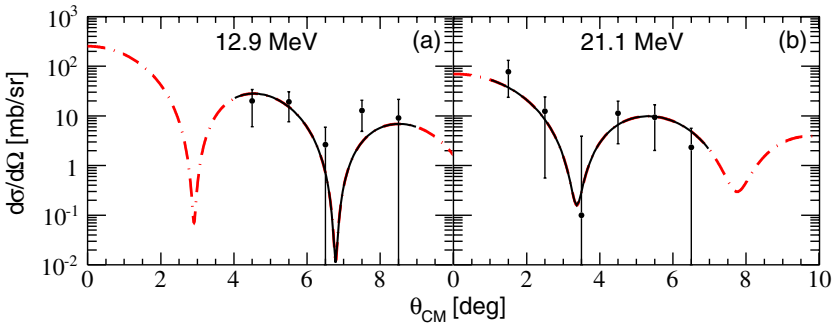

FIG. 3 (color online). Angular distributions for the modes located at $12.9 \mathrm{MeV}$ (a) and 21.1 MeV (b). The black solid line corresponds to the fit based on DWBA calculation using microscopic RPA predictions with isoscalar $L=0$ multipolarity. Theses predictions are represented in red dot-dashed line. 
were calculated using the same procedure as described above in the Lorentzian method. Theoretical angular distributions have been extracted from the DWBA calculations for thirteen excitation-energy intervals in steps of $2 \mathrm{MeV}$ and for isoscalar $L=0, L=1$ and $L=2$ excitations. After the background subtraction, experimental angular distributions were extracted for these excitationenergy intervals in steps of $2 \mathrm{MeV}$ and were fitted using a linear combination of these theoretical distributions. The coefficient for the $L=0$ contribution in this fit provides access to the isoscalar monopole strength in the ${ }^{68} \mathrm{Ni}$ excitation and this is displayed in Fig. 4. Due to a nonnegligible uncertainty on normalization, large uncertainties are obtained on the absolute value of the exhausted fraction of the EWSR in the MDA analysis. Therefore, only relative values of EWSR are given. In the shape of this spectrum, the isoscalar $L=0$ excitation has a large contribution in the [17 to $29 \mathrm{MeV}$ ] region, and a soft one around $14 \mathrm{MeV}$.

In MDA the mean energy is given by $m_{1} / m_{0}$, so the low-energy isoscalar monopole mode is located at 14.3 MeV, compared to $12.9 \pm 1.0 \mathrm{MeV}$ in Lorentzian method analysis, and the whole ISGMR at $23.4 \mathrm{MeV}$, compared to $21.1 \pm 1.9 \mathrm{MeV}$ in Lorentzian method analysis for the first part of the ISGMR. Considering the error bars on this previous result in Lorentzian method analysis, and the fact that the binning of $2 \mathrm{MeV}$ used in MDA can influence the value of $m_{1} / m_{0}$, both the Lorentzian method and MDA results are compatible for the centroid energies of the different modes. Around $21 \mathrm{MeV}$, the monopole components dominate, and around $14 \mathrm{MeV}$ the strength is shared between $L=0$ and $L=1$ contributions, this point will be detailed in a future paper. According to the monopole

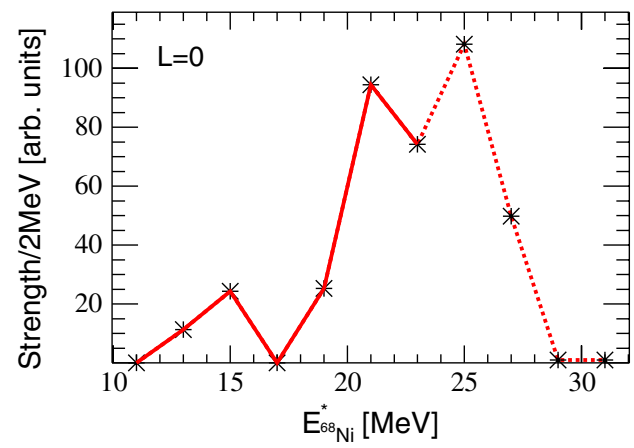

FIG. 4 (color online). Isoscalar monopole strength distribution extracted from MDA analysis. Large and small peaks are located at $m_{1} / m_{0}=23.4$ and $14.3 \mathrm{MeV}$, respectively. Several multipolarities contribute above $23 \mathrm{MeV}$ (red dotted line). The absolute value of the exhausted fraction of the EWSR is in arbitrary units, due to the uncertainty on normalization, the fragmentation of the strength and the emergence of soft monopole modes: the MDA is more model dependent than in the case of a well energy-localized single ISGMR, as in stable nuclei. strength distribution of Fig. 4, the width, of the lowenergy monopole mode is $0.7 \mathrm{MeV}$ and the width of the main ISGMR peak is $6.5 \mathrm{MeV}$ after deconvolution of resolution. The positions of the monopole modes are in good agreement with the predictions of relativistic and nonrelativistic RPA calculations [14]. These calculations indicate that the GMR is a pure isoscalar mode while the soft monopole mode is a pure neutron excitation. Similar conclusions can be drawn from examination of the transition densities used for the present data analysis: as displayed in Fig. 5, transition densities for protons and neutrons are in phase for the GMR [Fig. 5(a)], indicating an isoscalar mode, whereas only the neutron transition density is non-negligible for the soft monopole mode [Fig. 5(b)], indicating a neutron excitation.

In summary, the isoscalar monopole response has been measured in inverse kinematics in the nucleus ${ }^{68} \mathrm{Ni}$ with an intensity of $4 \times 10^{4}$ pps and using the active target MAYA. This is the first measurement of the monopole strength in a short-lived neutron-rich nucleus using inelastic alpha scattering. The ISGMR was found to be fragmented with a centroid at $21.1 \mathrm{MeV}$ and indications for a new type of excitation located around 13-14 MeV, the neutrondominated soft monopole mode, has been obtained. The present experimental method can be applied to a large domain of unstable and exotic nuclei that are already, or will be soon, available at rare isotope beam facilities worldwide. A next generation of active targets will provide access to smaller angle measurements and better energy and angular resolution. This shall permit studies of, for example, the fine structure of the soft monopole mode in order to provide quantitative information on the spin-orbit splitting. Measurements on heavier nuclei such as ${ }^{132} \mathrm{Sn}$ would also complement existing ISGMR systematics already established for stable $\mathrm{Sn}$ isotopes.

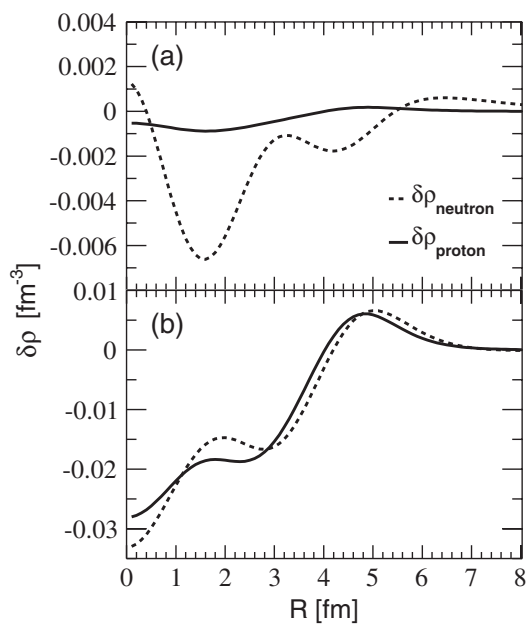

FIG. 5. (a) Proton (solid line) and neutron (dashed lines) ${ }^{68} \mathrm{Ni}$ monopole transition densities at $13 \mathrm{MeV}$, calculated with the RPA and SkI2 [33] interaction. (b) Same at $21 \mathrm{MeV}$. 
The authors thank M. Marqués for fruitful discussions. This work has been supported by Institut Universitaire de France and in part by the U. S. National Science Foundation (Grant No. PHYS-1068192). The research leading to these results has received funding from the European Union's Seventh Framework Program under Grant Agreement No. 262010.

*Present address: Grand Accélérateur National d'Ions Lourds (GANIL), CEA/DSM-CNRS/IN2P3, Bvd Henri Becquerel, 14076 Caen, France.

[1] O. Sorlin and M.-G. Porquet, Prog. Part. Nucl. Phys. 61, 602 (2008).

[2] M. N. Harakeh and A. van der Woude, Giant Resonances (Oxford University, New York, 2001).

[3] T. Li et al., Phys. Rev. Lett. 99, 162503 (2007).

[4] C. Monrozeau et al., Phys. Rev. Lett. 100, 042501 (2008).

[5] T. Li et al., Phys. Rev. C 81, 034309 (2010).

[6] D. Patel et al., Phys. Lett. B 718, 447 (2012).

[7] J. Piekarewicz, Phys. Rev. C 76, 031301 (2007).

[8] J. Li, G. Colò and J. Meng, Phys. Rev. C 78, 064304 (2008).

[9] E. Khan, Phys. Rev. C 80, 011307(R) (2009).

[10] P. Veselý, J. Toivanen, B. G. Carlsson, J. Dobaczewski, N. Michel, and A. Pastore, Phys. Rev. C 86, 024303 (2012).

[11] E. Khan, J. Margueron and I. Vidaña, Phys. Rev. Lett. 109, 092501 (2012).

[12] L.-G. Cao, H. Sagawa, and G. Colò, Phys. Rev. C 86, 054313 (2012).

[13] L. Capelli, G. Colò, and J. Li, Phys. Rev. C 79, 054329 (2009).

[14] E. Khan, N. Paar, and D. Vretenar, Phys. Rev. C 84, 051301 (2011).
[15] E. Khan, N. Paar, D. Vretenar, L.-G. Cao, H. Sagawa, and G. Colò, Phys. Rev. C 87, 064311 (2013).

[16] E. Yüksel, E. Khan and K. Bozkurt, Eur. Phys. J. A 49, 124 (2013).

[17] D. Savran, T. Aumann and A. Zilges, Prog. Part. Nucl. Phys. 70, 210 (2013).

[18] D. Vretenar, Y. F. Niu, N. Paar, J. Meng, Phys. Rev. C 85, 044317 (2012).

[19] O. Wieland et al., Phys. Rev. Lett. 102, 092502 (2009).

[20] D. M. Rossi et al., Phys. Rev. Lett. 111, 242503 (2013).

[21] R. Anne, in Proceedings of the International Symposium on Exotic Nuclei 2001, edited by Yu. E. Penionzhkevich and E. A. Cherepanov (World Scientific, Singapore, 2001), p. 634.

[22] C. E. Demonchy et al., Nucl. Instrum. Methods Phys. Res., Sect. A 573, 145 (2007).

[23] J. Pancin, J. Gibelin, M. Goth, P. Gangnant, J.-F. Libin, R. Raabe, T. Roger, and P. Roussel-Chomaz, JINST 7, P01006 (2012).

[24] T. Roger, M. Caamaño, C. E. Demonchy, W. Mittig, H. Savajols, and I. Tanihata, Nucl. Instrum. Methods Phys. Res., Sect. A 638, 134 (2011).

[25] J. F. Ziegler, J. Appl. Phys. 85, 1249 (1999).

[26] S. Agostinelli et al., Nucl. Instrum. Methods Phys. Res., Sect. A 506, 250 (2003).

[27] J. Pancin et al., Nucl. Instrum. Methods Phys. Res., Sect. A 735, 532 (2014).

[28] Y.-W. Lui, D. H. Youngblood, H. L. Clark, Y. Tokimoto, B. John, Phys. Rev. C 73, 014314 (2006).

[29] I. J. Thompson, Comput. Phys. Rep. 7, 167 (1988).

[30] J. Cook, Comput. Phys. Commun. 25, 125 (1982).

[31] J. Albiński et al., Nucl. Phys. A445, 477 (1985).

[32] G. Colò, L. G. Cao, N. Van Giai, L. Capelli, Comput. Phys. Commun. 184, 142 (2013).

[33] P. G. Reinhard and H. Flocard, Nucl. Phys. A584, 467 (1995). 